\title{
Diagnostic and therapeutic approach to children with Nijmegen breakage syndrome in relation to development of lymphoid malignancies
}

\author{
Aleksandra Filipiuk ${ }^{1, A-D \oplus}$, Agata Kozakiewicz ${ }^{1, B-D \oplus}$, Kamil Kośmider, ${ }^{1, B-D \oplus}$, Monika Lejman ${ }^{2, B-D \oplus}$, \\ Joanna Zawitkowska ${ }^{1, C-D, F \oplus}$ \\ ${ }^{1}$ Department of Paediatric Haematology, Oncology, and Transplantology, Medical University, Lublin, Poland \\ ${ }^{2}$ Laboratory of Genetic Diagnostics, Department of Paediatric Haematology, Oncology and Transplantology, Medical \\ University, Lublin, Poland \\ A - Research concept and design, B - Collection and/or assembly of data, C - Data analysis and interpretation, \\ $D$ - Writing the article, $E$ - Critical revision of the article, F - Final approval of the article
} Filipiuk A, Kozakiewicz A, Kośmider K, Lejman M, Zawitkowska J. Diagnostic and therapeutic approach to children with Nijmegen breakage
syndrome in relation to development of lymphoid malignancies. Ann Agric Environ Med. 2022; 29(2): 207-214. doi: 10.26444/aaem/143541

\begin{abstract}
Introduction and objective. Nijmegen breakage syndrome (NBS) is a rare chromosomal instability disorder. The majority of patients carry founder mutation in the NBN gene (c.657_661del5). Characteristic features of the NBS include progressive microcephaly, dysmorphic facial features, immunodeficiency, and high predisposition to malignancy with cumulative cancer incidence by the age of 20 years, and amounted to over $70 \%$.

The aim of study is to present the latest methods of diagnosis, potential cancer risk factors and treatment of lymphoid malignancies in children with NBS.

Review methods. To review the evidence using PubMed and Google Scholar search which included articles published between 2009-2021, focusing on articles published between 2013-2021.

Abbreviated description of the state of knowledge. The average delay in diagnosis of NBS ranges from 4-5 years. Neonatal screening of T-cell excision circles (TRECs) and kappa-deleting recombination excision circles (KRECs) seems favourable in NBS. There are no specific protocols for the treatment of lymphoid malignancies in children with NBS, and full- dose chemotherapy is the most frequently applied method. Reducing the doses of chemotherapy does not significantly reduce the toxicity. Main cause of death is cancer progression and treatment-related mortality mostly associated with infectious complications. Patients with diagnosed cancer who received haematopoietic stem cell transplantation (HSCT) had significantly higher 20 -year OS than those who did not (42.7\% vs. $30.3 \%)$.

Summary. Further meta-analysis is essential to establish the best monitoring and treatment regimen in patients with NBS and lymphoid malignancies.
\end{abstract}

\section{Key words}

diagnosis, lymphoma, antineoplastic agents, Nijmegen Breakage Syndrome, leukemia, Haematopoietic Stem Cell Transplantation

\section{INTRODUCTION}

Nijmegen breakage syndrome (NBS) is a rare chromosomal instability disorder. The gene responsible for the disease, $N B N$, is located on chromosome $8 \mathrm{q} 21$. The majority of patients are diagnosed with five base pair deletion in NBN gene (c.657_661del5) which is a founder mutation in Slavic populations [1]. NBS seems to occur worldwide, but most patients originated from Poland and Russia [2, 3].

The NBN gene codes for nibrin which is involved in DNA double-strand break repair [4]. Due to mutation, V(D)J rearrangement of $\mathrm{T}$ cells is affected and circulating $\mathrm{T}$ cells have signs of senescence in the young patients with NBS $[5,6]$.

The characteristic clinical features of the NBS include progressive microcephaly, dysmorphic facial features

Address for correspondence: Aleksandra Filipiuk, Department of Paediatric Haematology, Oncology, and Transplantology, Medical University, Lublin, Poland E-mail:aleksandrafili6@gmail.com

Received: 01.06.2021; accepted: 02.11.2021; first published: 18.11.2021 (including sloping forehead, prominent nose, long mandible, long philtrum), mild growth delay and premature ovarian insufficiency. Profound immunodeficiency both cellular and humoral response develops among most patients [4]. Patients with NBS have cumulative cancer incidence by the age of 20 years and amounted to over $70 \%[2,3]$. Non-Hodgkin lymphomas (NHLs) are most common with a similar number of lymphomas developed from abnormal T-cells and B-cells. T-cell acute lymphoblastic leukemia (T- ALL) is most frequently diagnosed among leukemias $[2,3,7,8,9]$.

\section{OBJECTIVE}

The aim of the study is to present the current state of knowledge on NBS and lymphoid malignancies with a focus on the peculiarities of diagnosis, cancer risk factors and treatment. 


\section{REVIEW METHODS}

To review the evidence, use was made of a PubMed and Google Scholar search on 30 June 2021 which included articles published between 2009-2021, focusing on articles published between 2013-2021. Combinations of the following key words were used: 'Nijmegen Breakage syndrome', 'diagnosis, 'TRECs' 'KRECs', 'primary immunodeficiency' 'cancer risk factors', 'lymphoid malignancies', 'chemotherapy', 'ECIL-8', 'HSCT', 'immunotherapy', 'second malignancies' (key words: Nijmegen Breakage syndrome, leukemia, lymphoma, diagnosis, Haematopoietic Stem Cell Transplantation, antineoplastic agents) to review the evidence. The articles were in English. After compiling a list of potentially relevant articles, the full text of each paper was appraised, with particular emphasis on articles presenting patients with Nijmegen Breakage syndrome and lymphoid malignancies. Due to the rarity of the disease, case reports have been also included in the review.

\section{STATE OF KNOWLEDGE}

Epidemiology of NBS. The disease likely occurs worldwide, but the highest occurrence of NBS has been described in Central and Eastern Europe due to the homozygous c.657_661del5 mutation in NBN gene. Most patients with NBS originate from Poland (137 patients), followed by Russia (81 patients), Ukraine (56 patients), the Czech Republic (33 patients), Belarus (22 patients) and Slovakia (14 patients) $[1,2,3]$. An unexpectedly high incidence of NBS patients has also been reported in regions of southeast Germany, which is linked to Slavic origin and migration in the Middle Ages [10]. Single cases of such a genetic disorder have also been reported in Turkey, the United Kingdom, the United States of America, Austria, and Macedonia [2, 11]. Overall, analysis shows the highest prevalence of NBS in Poland and the Czech Republic $[1,2]$. The manner of the reported prevalence can be explained that founder mutation c.657del 5 most likely occurred less than 300 generations ago, which confirms that the original mutation predated the historic split and subsequent spread of the 'Slavic people'. Moreover, the massive population movement after World War II influenced the geographical distribution of NBS [1]. Three patients with NBS have been described in Pakistan and are homozygous carriers of an other mutation c.1089C $>$ A [2].

Diagnosis of NBS. The diagnosis of NBS is based on clinical manifestations and is confirmed by genetic analysis. The hallmark symptom of NBS is progressive microcephaly, which influences facial phenotype. The sloping forehead, the small nose, the receding chin, and the relatively large ears are the most common. Clinical features are also mild growth retardation, general lack of neurological symptoms, recurrent respiratory tract infections and malignancies [4]. Median age at diagnosis of NBS ranged from 4-5 years. This is about average for all the countries [2,3]. Delay in diagnosis can result from the absence of unambiguous symptoms of NBS. There is no data on the most common symptoms at which diagnosis was started; notably, children with the mildest form of NBS remain underdiagnosed.

Microcephaly is a frequent clinical sign in various disease entities. The putative etiology for microcephaly is ascertained in $59 \%$ of all patients [12]. Deripapa et al. observed that microcephaly was diagnosed at birth or shortly thereafter in $80 \%$ patients with NBS; however, the average delay of NBS diagnosis was 6.5 years [8]. Wolska-Kuśnierz et al. reported that prevalence of sporadic respiratory tract infections differs slightly from the incidence of severe respiratory problems. The most frequent infections are pneumonia and acute bronchitis. Surprisingly, the incidence of opportunistic infections is low, affecting only about $5 \%$ [7]. The distribution of infections does not differ significantly from the general paediatric population and makes the decision about starting diagnosis more difficult. Patients born in large cities and the capitals of Eastern Slavic countries are diagnosed earlier in comparison to those from rural regions, which may be partly explained by accessibility of specialist medical care $[2,3]$.

For Slavic populations, sequencing of exon 6 of the NBN gene to detect founder mutation c.657_661del5 on both alleles is the method of choice $[2,3,4]$. Although genotyping common founder mutations in NBS is the basic diagnostic method, it results in delays in diagnosis. T-cell excision circles (TRECs) and kappa-deleting recombination excision circles (KRECs) can be potentially useful for the detection of NBS patients during neonatal screening [13]. Measurements of TRECs, KRECs and beta-actin as quality markers of DNA based on real-time polymerase chain reaction are used for screening severe primary immunodeficiency diseases (PID), mainly severe combined immunodeficiency. Routine screening is currently performed in the USA, Israel, New Zealand, Norway, Taiwan, several provinces in Canada, Switzerland, Iceland, Sweden, Italy (Tuscany), Spain (Catalonia), and in some regions in Australia [14]. In European countries, pilot projects have been announced in France, Spain, Norway, and the Netherlands [15]. In the USA, 2 cases of NBS were identified with solely low KRECs copy numbers [13], and 2 cases of NBS were reported with low TRECs [16, 17]. In the first population screening study for $\mathrm{T}$ and/or $\mathrm{B}$ immunodeficiency in Central and Eastern Europe, which included 44,287 newborns, one case of NBS with decreased values of TRECs and KRECs was identified [15].

Prophylaxis against viral, fungal, antibacterial and Pneumocystis jiroveci infections is not routinely recommended or implemented [7].

If there is lack of early diagnosis, children with NBS are exposed to ionizing radiation. Homozygous c.657-661del mutation affects cell radiosensitivity. Moreover, it is associated with disturbance in the activation of cell cycle checkpoints and with defects in DNA repair [18]. However, some patients were irradiated prior to NBS diagnosis with no unexpected toxicity or second tumour diagnosis [8].

Patients with undiagnosed NBS are exposed to adverse events related to immunization involving administration of viable vaccines and demand modifications in vaccination schedules. The association of immunodeficiency-related vaccine-derived rubella virus with cutaneous granulomatous disease has been reported. Granulomas are resistant to various types of therapy and have been resolved after haematopoietic stem cell transplantation (HSCT) in some patients with NBS [8, 19]. However, the use of attenuated BCG vaccine is favourable if the annual tuberculosis incidence is between $0.1-1 \%$, and none of NBS patients experienced any adverse effects $[7,20]$.

Factors of increased risk of lymphoid malignancies. Attempts are being made to find the causes of increased carcinogenesis. The MRE11, RAD50, and NBN gens encodes 
proteins of the MRN complex which is involved in various DNA damage repair pathways. Some specific alternations of the RAD 50 may be associated with childhood ALL [21]. Biallelic 657del 5 mutation of $N B N$ is associated with significant risk of cancers [22]. Interestingly, heterozygous carriers of mutation of the NBN gene have increased risk of development cancers, especially breast, prostate, and colorectal cancers, leukemias, and NHL. Among mutations in NBN gene, the 657 del5, the I171V and the R215W mutations are the most common [23]. However, the contribution of heterozygous p.I171V and p.R215W NBN mutations to cancer initiation is uncertain. The germline p.I171V may be considered as a risk factor in childhood ALL [21]. Significant differences in the number of chromatid breaks are observed only for mutant cells c. 657-661del, compared to control cells [24].

Chromosomal instability is associated with the development of complex genetic markers in pre-cancer cells. Despite the karyotype of NBS patients is generally normal, several abnormalities in the form of aneuploidies, structural rearrangements and marker chromosomes may be observed in $10-60 \%$ of cells [4].

Because of NBN mutation, T-cell development is affected at the stage of thymic output and $\mathrm{V}(\mathrm{D}) \mathrm{J}$ rearrangement. Circulating $\mathrm{T}$ cells of patients with NBS have signs of senescence which is already present in the youngest age group. Patients have a decreased level of CD4+ and CD8+ $\mathrm{T}$ cells. Although recent thymic emigrants and naïve $\mathrm{T}$ lymphocyte cell populations are significantly lower, the generation of antigen-primed (effector) T-cells is similar or even greater in NBS patients than in healthy control. There is high frequency of the more differentiated T cells expressing the senescent cell marker CD57, KLRG1, PD1 and do not express co-stimulatory molecule CD28 $[5,6]$. Such properties might be related to increased susceptibility to lymphoid malignancies.

The extremely high cancer incidence in NBS can be explained by impaired telomeric repair which synergize with telomere attrition. Alternatively, cultivated lymphoblastoid cells derived from NBS patients with long survival times ( $>12$ years) display shorter telomeres and lower caspase 7 activities, compared to cells derived from patients with short survival times ( $<3$ years), suggesting low apoptosis rates and increased senescence rates with effective tumour suppression. In view of the bifunctional role of telomere attrition, an unambiguous role in carcinogenesis is difficult to identify [25].

Viral infections and monoclonal gammopathy are analyzed for early detection of changes preceding the development of malignancy. The predominance of EBV and monoclonal gammopathy, mainly of the IgM type, accompanied by an elevated level of IgM, are most often reported in patients who developed B-NHL. Clonal Ig (IgH, IgK or IgL) and/or TCR (BG and/or D) gene rearrangements are observed in $73.9 \%$ of patients and precede the diagnosis of lymphoma, on average, by 3 years. However, the strength of these associations needs to be further evaluated [26].

Another cause that directly affects carcinogenesis is chronic oxidative stress which is caused by endogenous reactive oxygen species overproduction and impairment of mitochondrial homeostasis. Oxidative stress leads to structural changes in DNA, proteins, and lipids [27]. Maciejczak et al. reported only significantly increased activity of catalase, decreased total antioxidant capacity and greater oxidative damage to proteins (advanced glycation end products, advanced oxidation protein products), lipids (4-hydroxynonenal protein adducts, 8-isoprostanses) and DNA (8-hydroxy2'deoxyguanosine) [28]. Patients with NBS display statistically lower total antioxidant status. CoQ10, which deficiency of which may contribute to abnormal function of mitochondria during cancer, is significantly lower. There is a need for diagnosing oxidative stress as a potential disease biomarker. Supplementation with tocopherol and CoQ10 may enhance treatment efficacy in NBS diseases [29].

In literature, the search for cancer prevention is made in NBS patients. Antisense oligonucleotides as a potential cancer prophylaxis have been demonstrated. Antisense oligonucleotides have been used to enforce alternative splicing in humanized NBS murine mouse model to remove exon 6 and 7 , and efficiently generate $\mathrm{p}-80$ protein. This $\mathrm{p} 80$-nibrin contributes to a milder phenotype and improves survival [30].

Characteristic of tumours in NBS patients. The cumulative cancer incidence by the age of 10 years amounted to $41.6 \%$ [3] and $40.21 \%$ [2], and dramatically increased further to $71.6 \%$ [3] and $77.78 \%$ [2] by the age of 20 years. The first episode of malignancy occurred at a median age of 6.0-10.7 years. Male predominance is observed in patients with cancers and NBS $[2,3,7,9,31]$, with the exception of one study with female predominance [8]. With respect to tumour type, NHLs represent most malignancies with a similar number of lymphoid tumours of B-cell origin and lymphomas developed from abnormal T-cells. Diffuse large B-cell lymphoma (DLBCL) was the most common among B-cell lymphomas and T- cell lymphoblastic lymphoma (T-LBL) among T-cell lymphomas. Interestingly, peripheral T-cell lymphomas (PTCL) are diagnosed in patients with NBS, which are tumours rarely diagnosed in the non-syndromic paediatric population [32]. Most patients are classified as stage III or IV. T-ALL is most often diagnosed in acute leukemia. Most patients are classified as high-risk group. Among other types of solid malignancies, there were diagnosed medulloblastoma, neuroblastoma, thyroid carcinoma, gastric carcinoma, lowgrade glioma, rhabdomyosarcoma, unspecified liver tumour, dysgerminoma and ganglioglioma [2, 3, 7, 33]. Data on neoplasms in NBS patients are presented in Table 1.

Treatment strategies of lymphoid malignancies in NBS patients. There are no specific protocols for the treatment of lymphoid malignancies in children with NBS. The decision to reduce cytostatic agents depends on the oncologist and is based on the detailed medical history of every patient. Fulldose chemotherapy is the most frequently applied method $[3,8,21]$. In previous studies, it was suggested to administer $80 \%$ of dose chemotherapy, particularly alkylating agents as cyclophosphamide, DNA topoisomerase II, inhibitors, and antimetabolites such as methotrexate, and increase doses when well toleration is observed $[11,34]$. Similarly, Aleinikova et al. reported that unrecognized NBS is the main reason for non-reduced dose regimens during induction therapy, and can be linked with increased toxicity of chemotherapy [ 9 , 35]. Interestingly, Schutte et al. reduced over $25 \%$ of doses of chemotherapy agents, methotrexate, daunorubicin, doxorubicin, cyclophosphamide; however, severe adverse effects were observed [33].

Patients with a decreased immunoglobulins level should receive intravenous immunoglobulins (IVIG). According to current guidelines for immunoglobulin supplementation in 
Table 1. Types of neoplasms in patients with NBS

\begin{tabular}{|c|c|c|c|c|c|c|}
\hline References & $\begin{array}{c}\text { Wolska-Kuśnierz } \\
\text { (2015) [7] }\end{array}$ & $\begin{array}{l}\text { Aleinikova } \\
(2015)[9]\end{array}$ & $\begin{array}{l}\text { Attarbaschi } \\
(2016)[31]\end{array}$ & $\begin{array}{l}\text { Deripapa } \\
\text { (2017) [8] }\end{array}$ & $\begin{array}{c}\text { Wolska-Kuśnierz } \\
\text { (2020) [2] }\end{array}$ & $\begin{array}{c}\text { Sharapova } \\
(2021) \text { [3] }\end{array}$ \\
\hline No. of patients with NBS & 149 & 19 & - & 35 & 241 & 136 \\
\hline Median age (years) at diagnosis & 10.25 & 10.7 & 9.95 & 6.0 & 9.31 & - \\
\hline B-NHL & 30 & - & 12 & 11 & 49 & 20 \\
\hline DLBCL & - & - & 8 & 10 & 25 & 16 \\
\hline T-NHL & 21 & 7 & 11 & 4 & 46 & 18 \\
\hline T-LBL & - & - & 5 & 2 & 43 & 13 \\
\hline PTCL & - & - & 6 & 2 & 3 & 5 \\
\hline $\mathrm{ALCL}$ & - & - & 1 & - & 2 & 1 \\
\hline Other NHL & - & 1 & 2 & - & - & 3 \\
\hline $\mathrm{HL}$ & 7 & - & - & - & 9 & 2 \\
\hline T-ALL & 10 & - & - & - & 23 & 9 \\
\hline BCP-ALL & - & - & - & - & 3 & 1 \\
\hline AML & - & - & - & - & 1 & 1 \\
\hline other & 7 & 1 & - & 1 & 5 & 1 \\
\hline Other solid malignancies & 5 & - & - & 1 & 11 & 4 \\
\hline
\end{tabular}

- no data; NBS-Nijmegen breakage syndrome; B-NHL-B-cell non-Hodgkin lymphoma; DLBCL-diffuse large B-cell lymphoma; T-NHL-T-cell non-Hodgkin lymphoma; T-LBL-T-cell lymphoblastic lymphoma; PTCL - peripheral t-cell lymphoma; ALCL - anaplastic large cell lymphoma; HL - Hodgkin lymphoma; AL - acute leukemia; T-ALL - T-cell acute lymphoblastic leukaemia; BCP-ALL - B-cell progenitor acute lymphoblastic leukemia; AML - acute myeloid leukemia.

primary immunodeficiencies, NBS patients should receive IVIG to maintain a serum IgG level over $5.0 \mathrm{~g} / \mathrm{l}$ [36]. However, Deripapa et al. postulate that most (if not all) patients require IVIG substitution, and this decision should be made based on antibody-specific response. The ECIL-8 (European Conference on Infections in Leukaemia) group does not recommend routine antibacterial prophylaxis for patients at high risk, which contrasts with a previous international paediatric specific guideline. However, careful risk-benefit evaluation might favour antibacterial prophylaxis in individual patients, depending on their circumstances [37]. Due to musculoskeletal problems and central nervous system adverse events (CNS-AEs), the European Medicines Agency issued warning against the use of fluoroquinolones [37]. The previously recommend short time of oral prophylaxis in most immunodeficient patients, should not be implemented [36]. Reduction of invasive fungal infections is crucial for reduction of mortality during chemotherapy in NBS patients. Therefore, consistently with ECIL-8, fluconazole and posaconazole in patients over the age of 13 years older should be administered [38].

There is limited data on the frequency of toxicities in NBS patients with malignancies. In the Belarusian experience, all patients on chemotherapy experienced grade 3-4 complications according to the National cancer Institute Common Toxicity Criteria. The most common were neutropenic infections and haemostatic disorders $[9,35,39]$.
Zawitkowska et al. reported that complications occur mostly in the induction phase of therapy, and in the reinduction phase. Among the analyzed toxicities in children with ALL, the most common included infection, gastrointestinal toxicity, and hepatotoxicity [40]. Dexamethasone during the induction phase of EURO-LB 02 may prevent central nervous system relapse more efficiently than prednisone, but produces significantly more grade 3 and 4 toxicities [41].

The main cause of death is cancer progression and treatment-related mortality, mostly associated with infectious complications $[2,3,7,8,9,31]$.

The overall survival rates for patients with DNA damage repair defect and without any cancer susceptibility syndromes are $9.7 \%$ and $68.7 \%$, respectively [42]. Mortality rate in patients with NBS and malignancies ranges from $22.6 \%$ $67.6 \%$ (Tab. 2). This divergence might be explained by delivery of full- dose chemotherapy along with IVIG treatment $[7,8]$. The next contributing factor might be supplementation of immunoglobulins, irrespective of IgG levels. Low IgG levels were observed as a laboratory predictor of severe infections which is related to poor prognosis [7]. KRECs numbers are also reliable predictors of severe infections, oncological, and possible autoimmune complications [8]. TRECs has been reported as indicator of thymic output of naïve T-cells [5]; however, Deripapa et al. observed no correlation between TRECs and severity of infections. Moreover, complex aberrations related to chromosomal instability which are

Table 2. Outcome of patients with Nijmegen breakage syndrome and lymphoid malignancies

\begin{tabular}{|c|c|c|c|c|c|}
\hline & $\begin{array}{l}\text { Wolska- Kuśnierz } \\
\text { (2015) [7] }\end{array}$ & $\begin{array}{l}\text { Attarbaschi } \\
(2016)[31]\end{array}$ & $\begin{array}{l}\text { Deripapa } \\
\text { (2017) [8] }\end{array}$ & $\begin{array}{l}\text { Wolska-Kuśnierz } \\
\text { (2020) [2] }\end{array}$ & $\begin{array}{c}\text { Sharapova } \\
(2021)[3]\end{array}$ \\
\hline No. of patients with malignancies & 63 & 26 & 20 & 151 & 62 \\
\hline $\mathrm{CR}$ & - & $10(38 \%)$ & - & - & $35(71.4 \%)$ \\
\hline Relapse/progression & - & $10(38 \%)$ & $5(25 \%)$ & - & $12(24.5 \%)$ \\
\hline Death & $28(44 \%)$ & $16(62 \%)$ & $5(25 \%)$ & $101(67.6 \%)$ & $14(22.6 \%)$ \\
\hline
\end{tabular}


revealed in cytogenic karyotype and microarray tests, trigger poor outcome [43]. The next factor for poor prognosis is not performing a transplantation [8].

About a quarter of patients with NBS have relapse [3, 8]. Tomasik et al. observed that heterozygous carriers of the c.657_661del 5 mutation in the NBN gene are at higher risk of BCP-ALL relapse within the CNS, compared to NBS patients [44].

Possibilities of molecular targeted therapy for children with NBS. Considering the poor outcome of patients with NBS and malignancies, new strategies of therapy are still needed to reduce clonality of lymphocytes. There are limited data on the use of molecular targeted therapy in the treatment of children with PIDs and lymphoid malignancies. Therapeutic approach should be considered in relation to the nature of the NBS as a DNA repair disorder, and to the remaining immune function available in the patient's body, which is required to enhance immunotherapy. Antitumoural targeted therapies, such as monoclonal antibodies (mAb), chimeric antigen receptor (CAR) T-cells and small-molecule inhibitors, potentially offer significant opportunities for optimizing treatment of lymphoid malignancies $[45,46]$.

Monoclonal antibodies have shown great advances since their first member rituximab in 1997, respectively. The next-generation of antibodies aim to boost antibodydependent cellular cytotoxicity and component-dependent cytotoxicity, for example, obinutuzumab. MAbs may also be combined with antineoplastic drugs intensifying its concentration in tumour cells, such as gemtuzumab ozogamicin. Bi-specific T-cell engaging (BiTE) Abs recruit polyclonal $\mathrm{T}$ lymphocyte population, avoiding the need for antigen specificy and putative tumour immune evasion through HLA downregulation. MAbs can also interfere with immune checkpoint pathways and allow potentiate anti-tumour responses, for instance, pembrolizumab -antiprogrammed cell-death 1 - PD1. The CAR T-cells, which are autologous T-cells from patients and genetically engineered to express tumor-reactive CARs, are the most recent method of treatment $[46,47]$. The next group are inhibitors of PI3K/
$\mathrm{AKT} / \mathrm{mTOR}$ pathway, such as sirolimus. In EBV- driven lymphoid malignancies anti-EBV cytotoxic T-lymphocytes are considered as a salvage therapy in refractory disease [45]. There are reported cases that therapy was well tolerated in patients with ataxia telangiectasia [48].

Therapies that target cell surface antigens are currently undergoing clinical trials with most common indication for refractory/relapse lymphoid malignancy [46]. Agents that may potentially be used in the treatment of patients with NBS and lymphoid malignances are listed in Table $3[45,46,47]$. There are no supportive data on the use of inhibitors of the PI3K/ $\mathrm{AKT} / \mathrm{mTOR}$ pathway in patients with DNA repair defects [45].

Referring to the early age of cancer development in NBS, there are data on blinatumomab in the treatment of children aged one year or older [46]. Patients with NBS and leukemias are a potential group among other PIDs who can benefit from treatment with blinatumomab due to the persistent residual function of T cells [45]. However, the use of molecular targeted therapies in patients with NBS has some limitations due to the nature of immunodeficiency. Reinfusing genetically engineered autologous $\mathrm{T}$ lymphocytes prove challenging, but it might be overcome by the development of universal allogeneic CAR T-cells derived from healthy donors [49]. Compared blinatumomab with CAR T-cells, blinatumomab has a few advantages: it is an off-the shelf product; it is less expensive, and the short half-life enables the precise control of serum levels [50].

Despite promising outcomes of targeted therapies, patients should be carefully observed due to potential toxicities. Invasive fungal disease is common in patients treated with blinatumomab, gemtuzumab ozogamicin and tisagenlecleucel, and uncommon in patients treated with brentuximab vedotin and pembrolizumab [46]. These data are particularly important for patients with immunodeficiencies and highlight the need to intensify antifungal prophylaxis. Cytokine release syndrome (CRS) is another toxicity, and many patients require administration of tocilizumab, an anti-interleukin 6 receptor antagonist [51]. Neurotoxicity after blinatumomab cannot be ignored in patients with NBS and prone to neurovegetative disorders $[45,52]$.

Table 3. Possibilities of molecular targeted therapy for children with NBS [45-47]

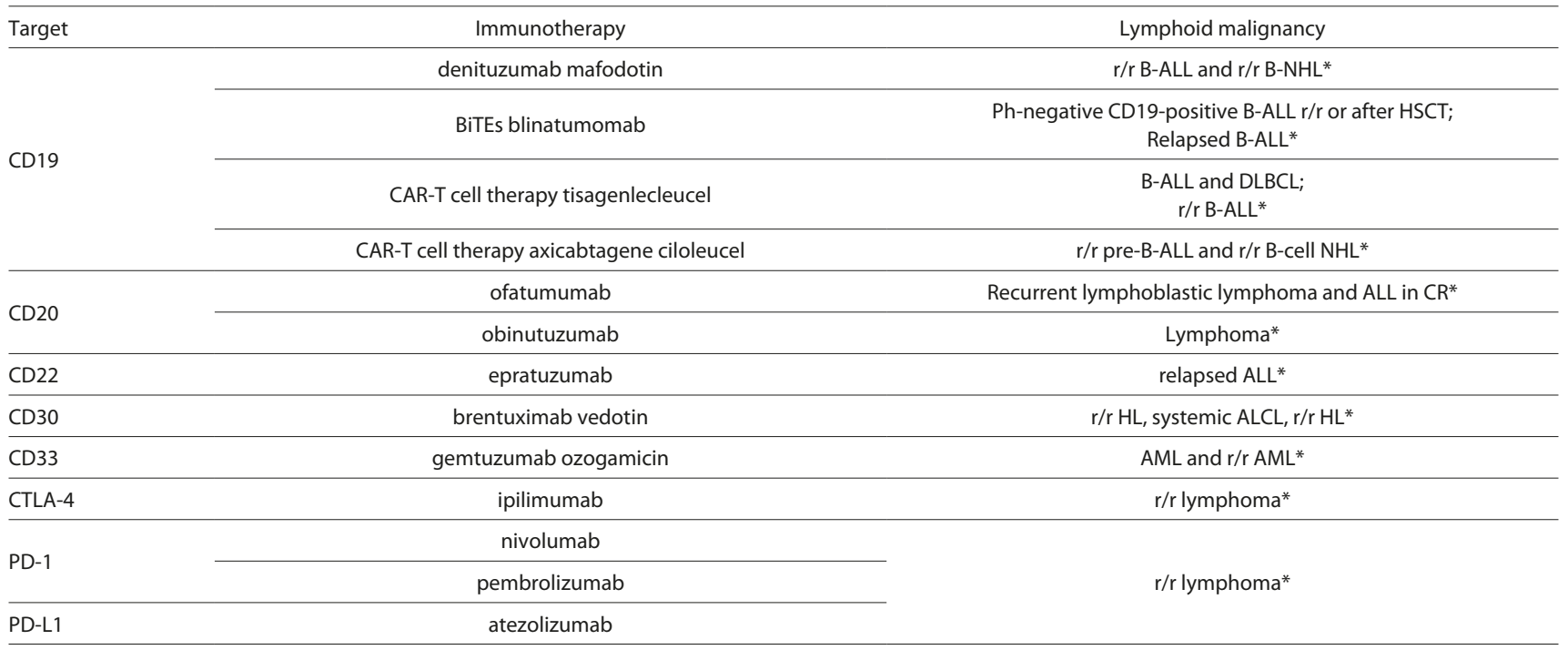

*ongoing clinical trial. NBS - Nijmegen breakage syndrome; CD - cluster of differentiation; ALL - Acute Lymphoblastic Leukemia; NHL - non-Hodgkin lymphoma; HSCT - Hematopoietic Stem Cell Transplantation; Ph - Philadelphia chromosome; DLBCL - diffuse large B-cell lymphoma; C - complete remission; HL - Hodgkin lymphoma; $\mathbf{A L C L}$ - anaplastic large cell lymphoma; AML - Acute Myeloblastic Leukemia; $\mathbf{r} / \mathbf{r}$ - relapsed or refractory; CTLA-4 - cytotoxic T-lymphocyte-associated protein 4; PD-1 - programmed cell death protein 1; PD-L1 - programmed death-ligand 1 protein 
Table 4. Characteristics of patients with NBS who underwent HSCT

\begin{tabular}{lccc}
\hline & Wolska- Kuśnierz (2015) [7] & Deripapa (2017) [8] & Wolska-Kuśnierz (2020) [2] \\
\hline No. of patients with NBS & 149 & 35 & 241 \\
\hline No. of patients with HSCT & $14(9.4 \%)$ & $15(42.3 \%)$ & $49(20.3 \%)$ \\
\hline Median age of HSCT & 8.8 & - & 136 \\
\hline HSCT due to malignancy & $11(78.6 \%)$ & $8(53.3 \%)$ & $35(71.4 \%)$ \\
\hline HSCT due to immunodeficiency & $3(21.4 \%)$ & $5(33.3 \%)$ & $14(28.6 \%)$ \\
\hline Death from relapse or infections & $5(35.7 \%)$ & $2(13.3 \%)$ & $13(26.5 \%)$ \\
\hline
\end{tabular}

- no data NBS - Nijmegen breakage syndrome

HSCT - haematopoietic stem cell transplantation

HSCT in NBS patients. The general reasons of HSCT are defined as infection (severe or recurrent infections), malignancy, bone marrow failure (defined as leukopenia, anaemia, or thrombocytopenia, without the presence of infection or malignancy), autoimmunity or pre-emptive [53].

Transplantation in NBS patients is recommended in first complete remission (CR) of lymphoma or leukemia. Some patients were transplanted after secondary tumours. Another indication was clinically relevant immunodeficiency with severe or chronic infections, or immune dysregulation [2]. Patients with NBS who underwent HSCT are presented in Table 4 . The reasons why not all patients have transplantations are the low percentage of patients who achieve complete remissions and the relatively high mortality due to infections during chemotherapy. On the other hand, there is disproportion between the severity of the infection's symptoms and underlying immunodeficiency. Research is needed to validate the transplantation in asymptomatic patients with NBS $[2,3]$.

Despite the immunodeficiency, rejection and poor stem cell engraftment are likely without preparative conditioning. There are myeloablative conditioning with alkylating agents or total body irradiation and reduced intensity conditioning (RIC) without alkylating agents and with fludarabine $\leq 150 \mathrm{mg} / \mathrm{m}^{2}$ and cyclophosphamide $\leq 40 \mathrm{mg} / \mathrm{kg}$ [53]. The use of an RIC in comparison to a myeloablative conditioning regimen demonstrates beneficial HSCT outcomes in patients with NBS. Sharapova et al. reported a patient who received myeloablative conditioning but died soon after HSCT [3]. Most patients received modified Fanconi RIC based on fludarabine, and cyclophosphamide with or without antithymocyte globulin therapy $[2,3,53,54]$. Laberko et al. reported that a conditioning regimen with treosulfan $30 \mathrm{~g} / \mathrm{m}^{2}$ demonstrated a low level of early transplanted-associated toxicity, and enhanced graft function with stable donor chimerism in comparison to regimen with busulfan [55].

In the literaturthe incidence of acute and chronic graft versus host disease (GvHD) is inconclusive, showing a higher rate of acute GvHD. Sharapova et al. observed acute GvHD in $38.5 \%$ patients [3]. However, there are reports that none of patients developed GvHD $[8,48]$. This does not seem to be related with use of matched, unrelated donors and the presence of malignancies. The most common non-GvHD complication is viraemia due to adenovirus, cytomegalovirus, Epstein-Barr virus, and BK virus. Visceral toxicity is rarely observed in NBS patients [2]. There is a report on a patient who developed choledocholithiasis and recto-sigmoid ulcers [56]. Some patients fully rejected the graft [8]. Fatal infections after HSCT are the most common cause of death. The next causes of death after HSCT are cancer progression and relapses, particularly of primary lymphomas $[2,3,7,8]$. Patients who undergo pre-emptive transplantation had a more favorable outcome. Wolska-Kuśnierz et al. reported no transplant-related deaths in this group [2]. Sharapova et al. observed that $67 \%$ of patients survived [3].

In a cohort study, a 20-year OS in a transplanted group did not significantly differ from that observed in the non-transplanted group. However, NBS patients with diagnosed cancer who received HSCT had a significantly higher 20 -year OS than those who did not. (42.7\% vs. 30.3\%) [2]. Similarly, Sharapova et al. reported that from the time of NBS diagnosis, the probability of 15 -year OS in patients who underwent HSCT is significantly highe, in contrast to those who did not undergo HSCT. (61.5\% vs 36.9\%) [3]. Despite the favourable outcome of patients who undergo HSCT due to malignancies, there is a need for the further monitoring of HSCT as a pre-emptive therapeutic approach. Cancer-free survival outcome should be evaluated in these patients.

Second malignancies in NBS patients. Second malignant neoplasm (SMN) is defined as a malignancy which occurs more than 5 years from diagnosis of the first malignancy among patients who achieved complete remission. Frequency of patients with NBS who developed subsequent malignancy ranges from $13.2 \%-19 \%$. However, it includes neoplasms which occur less than 5 years from the first diagnosis of malignancy, which makes this statistic less comparable with the other $[2,3,31]$. The median age of diagnosis is 18 years [2] and the most frequent second malignancies are lymphomas [2, 3, 7]. Wolska-Kuśnierz et al. confirmed T-cell leukemia / lymphoma as half of secondary tumours [2] The most common at primary diagnosis were B-cell lymphoma / leukemia with DLBCL as most frequent [2, 3, 31]. The general risk factors for the development of second neoplasms are acute-lymphoblastic-leukemia-type therapy, cumulative anthracycline dose, CNS-involvement, female gender, and cancer-predisposing conditions [57].

On the other hand, reduced chemotherapy does not prevent the development of SMN [2]. Patients with NBS have a higher risk of SMN, compared to other pre-existing disorders, but much less than patients with constitutional mismatch repair disease $[31,58]$. In the general population, Attarbaschi et al. reported that lymphoid malignancies are the most frequent SMN; however, Moser et al. observed predominantly carcinomas $[57,58]$.

The survival rate 10 years after diagnosis of SMN is $47 \%$. Patients with a known cancer predisposing syndrome have a much lower survival rate of only $11.1 \%$ [57]. The majority of patients with NBS and SMN die due to tumour progression and infectious complications during chemotherapy. In the 
literature, there are reports of patients who were successfully transplanted and remained in the remission of cancer for more than 5 years. Analysis of the impact of HSCT on the cumulative risk of death in the presence of secondary malignancy as a competing event, showed that the risk of death was reduced twofold in transplanted patients compared with non-transplanted individuals [2].

\section{CONCLUSIONS}

Further meta-analysis is essential to establish the best monitoring and treatment regimen in patients with NBS and lymphoid malignancies. TRECs and KRECs can be potentially useful for the detection of NBS patients during neonatal screening. Oxidative stress should be diagnosed as potential disease marker. Supplementation with tocopherol and CoQ10 may enhance treatment efficacy in NBS diseases. Patients with NBS and lymphoid malignancies are particularly vulnerable to a high incidence progression of disease and therapy-associated toxicity. However, full-dose chemotherapy is the most frequently applied method. HSCT has a significant positive effect on survival.

\section{REFERENCES}

1. Seemanova E, Varon R, Vejvalka J, et al. The Slavic NBN Founder Mutation: A Role for Reproductive Fitness?. PLoS One. 2016; 11(12): e0167984. https://doi.org/10.1371/journal.pone.0167984

2. Wolska-Kusnierz B, Pastorczak A, Fendler W, et al. Hematopoietic Stem Cell Transplantation Positively Affects the Natural History of Cancer in Nijmegen Breakage Syndrome. Clin Cancer Res. 2021; 27(2): 575-584. https://doi.org/10.1158/1078-0432.CCR-20-2574

3. Sharapova SO, Pashchenko OE, Bondarenko AV, et al. Geographical Distribution, Incidence, Malignancies, and Outcome of 136 Eastern Slavic Patients With Nijmegen Breakage Syndrome and NBN Founder Variant c.657_661del5. Front Immunol. 2021; 11: 602482. https://doi. org/10.3389/fimmu.2020.602482

4. Chrzanowska KH, Gregorek H, Dembowska-Bagińska B, Kalina MA, Digweed M. Nijmegen breakage syndrome (NBS). Orphanet J Rare Dis. 2012; 7: 13. https://doi.org/10.1186/1750-1172-7-13

5. Meijers RWJ, Dzierzanowska-Fangrat K, Zborowska M, et al. Circulating T Cells of Patients with Nijmegen Breakage Syndrome Show Signs of Senescence [published correction appears in J Clin Immunol. 2018 Apr 23]. J Clin Immunol. 2017; 37(2): 133-142. https:// doi.org/10.1007/s10875-016-0363-5

6. Piatosa B, Wolska-Kuśnierz B, Tkaczyk K, et al. T Lymphocytes in Patients With Nijmegen Breakage Syndrome Demonstrate Features of Exhaustion and Senescence in Flow Cytometric Evaluation of Maturation Pathway. Front Immunol. 2020; 11: 1319. https://doi. org/10.3389/fimmu.2020.01319

7. Wolska-Kuśnierz B, Gregorek H, Chrzanowska K, et al. Nijmegen Breakage Syndrome: Clinical and Immunological Features, LongTerm Outcome and Treatment Options - a Retrospective Analysis. J Clin Immunol. 2015; 35(6): 538-549. https://doi.org/10.1007/s10875015-0186-9

8. Deripapa E, Balashov D, Rodina Y, et al. Prospective Study of a Cohort of Russian Nijmegen Breakage Syndrome Patients Demonstrating Predictive Value of Low Kappa-Deleting Recombination Excision Circle (KREC) Numbers and Beneficial Effect of Hematopoietic Stem Cell Transplantation (HSCT). Front Immunol. 2017; 8: 807. https://doi. org/10.3389/fimmu.2017.00807

9. Aleinikova O, Fedorova A, Sharapova S. Should Allogeneic Hematopoietic Stem Cell Transplantation be a Treatment Option for Patients with Nijmegen Breakage Syndrome? Belarusian Experience. Cell Ther Transplant. 2015; 4(1-2): 31-37. https://doi.org/10.18620/18668836-2015-4-1-2-31-37

10. Maurer MH, Hoffmann K, Sperling K, Varon R. High prevalence of the NBN gene mutation c.657-661del5 in Southeast Germany. J Appl Genet. 2010; 51(2): 211-214. https://doi.org/10.1007/BF03195730
11. Kocheva SA, Martinova K, Antevska-Trajkova Z, Coneska-Jovanova B, Eftimov A, Dimovski AJ. T-lymphoblastic leukemia/lymphoma in macedonian patients with Nijmegen breakage syndrome. Balkan J Med Genet. 2016; 19(1): 91-94. https://doi.org/10.1515/bjmg-2016-0012

12. von der Hagen M, Pivarcsi M, Liebe J, et al. Diagnostic approach to microcephaly in childhood: a two-center study and review of the literature. Dev Med Child Neurol. 2014; 56(8): 732-741. https://doi. org/10.1111/dmcn.12425

13. Borte S, von Döbeln U, Fasth A, et al. Neonatal screening for severe primary immunodeficiency diseases using high-throughput triplex real-time PCR. Blood. 2012; 119(11): 2552-2555. https://doi.org/10.1182/ blood-2011-08-371021

14. van der Burg M, Mahlaoui N, Gaspar HB, Pai SY. Universal Newborn Screening for Severe Combined Immunodeficiency (SCID). Front Pediatr. 2019; 7: 373. https://doi.org/10.3389/fped.2019.00373

15. Giżewska M, Durda K, Winter T, et al. Newborn Screening for SCID and Other Severe Primary Immunodeficiency in the PolishGerman Transborder Area: Experience From the First 14 Months of Collaboration. Front Immunol. 2020; 11: 1948. https://doi.org/10.3389/ fimmu.2020.01948

16. Patel JP, Puck JM, Srinivasan R, et al. Nijmegen breakage syndrome detected by newborn screening for $\mathrm{T}$ cell receptor excision circles (TRECs). J Clin Immunol. 2015; 35(2): 227-233. https://doi.org/10.1007/ s10875-015-0136-6

17. Puck JM. Newborn screening for severe combined immunodeficiency and T-cell lymphopenia. Immunol Rev. 2019; 287(1): 241-252. https:// doi.org/10.1111/imr.12729

18. Patel JP, Puck JM, Srinivasan R, et al. Nijmegen breakage syndrome detected by newborn screening for $\mathrm{T}$ cell receptor excision circles (TRECs). J Clin Immunol. 2015; 35(2): 227-233. https://doi.org/10.1007/ s10875-015-0136-6

19. Buchbinder D, Hauck F, Albert MH, et al. Rubella Virus-Associated Cutaneous Granulomatous Disease: a Unique Complication in Immune-Deficient Patients, Not Limited to DNA Repair Disorders. J Clin Immunol. 2019; 39(1): 81-89. https://doi.org/10.1007/s10875018-0581-0

20. Bonilla FA. Update: Vaccines in primary immunodeficiency. J Allergy Clin Immunol. 2018; 141(2): 474-481. https://doi.org/10.1016/j. jaci.2017.12.980

21. Mosor M, Ziółkowska-Suchanek I, Nowicka K, Dzikiewicz-Krawczyk A, Januszkiewicz-Lewandowska D, Nowak J. Germline variants in MRE11/RAD50/NBN complex genes in childhood leukemia. BMC Cancer. 2013; 13: 457. https://doi.org/10.1186/1471-2407-13-457

22. Gao P, Ma N, Li M, Tian QB, Liu DW. Functional variants in NBS1 and cancer risk: evidence from a meta-analysis of 60 publications with 111 individual studies. Mutagenesis. 2013; 28(6): 683-697. https://doi. org/10.1093/mutage/get048

23. di Masi A, Berardinelli F, Lli DE, Antoccia A. Cancer proneness in Nijmegen Breakage Syndrome Carriers. In: Neri Ch, editor. Advances in Genome Science. Probing Intracellular Regulation; 2013. p. 101-119

24. Dzikiewicz-Krawczyk A, Mosor M, Januszkiewicz D, Nowak J. Impact of heterozygous c.657-661del, p.I171V and p.R215W mutations in NBN on nibrin functions. Mutagenesis. 2012; 27(3): 337-343. https://doi. org/10.1093/mutage/ger084

25. Habib R, Kim R, Neitzel H, et al. Telomere attrition and dysfunction: a potential trigger of the progeroid phenotype in nijmegen breakage syndrome. Aging (Albany NY). 2020; 12(12): 12342-12375. https://doi. org/10.18632/aging.103453

26. Gregorek H, Chrzanowska KH, Dzierzanowska-Fangrat K, et al. Nijmegen breakage syndrome: Long-term monitoring of viral and immunological biomarkers in peripheral blood before development of malignancy. Clin Immunol. 2010; 135(3): 440-447. https://doi. org/10.1016/j.clim.2010.01.008

27. Maciejczyk M, Mikoluc B, Pietrucha B, et al. Oxidative stress, mitochondrial abnormalities and antioxidant defense in Ataxiatelangiectasia, Bloom syndrome and Nijmegen breakage syndrome. Redox Biol.2017; 11:375-383. https://doi.org/10.1016/j.redox.2016.12.030

28. Maciejczyk M, Heropolitanska-Pliszka E, Pietrucha B, et al. Antioxidant Defense, Redox Homeostasis, and Oxidative Damage in Children With Ataxia Telangiectasia and Nijmegen Breakage Syndrome. Front Immunol. 2019; 10: 2322. https://doi.org/10.3389/fimmu.2019.02322

29. Pietrucha B, Heropolitanska-Pliszka E, Maciejczyk M, et al. Comparison of Selected Parameters of Redox Homeostasis in Patients with AtaxiaTelangiectasia and Nijmegen Breakage Syndrome. Oxid Med Cell Longev. 2017; 2017: 6745840. https://doi.org/10.1155/2017/6745840

30. Salewsky B, Hildebrand G, Rothe S, et al. Directed Alternative Splicing in Nijmegen Breakage Syndrome: Proof of Principle Concerning Its 
Therapeutical Application. Mol Ther. 2016; 24(1): 117-124. https://doi. org $/ 10.1038 / \mathrm{mt} .2015 .144$

31. Attarbaschi A, Carraro E, Abla O, et al. Non-Hodgkin lymphoma and pre-existing conditions: spectrum, clinical characteristics and outcome in 213 children and adolescents. Haematologica. 2016; 101(12): 1581-1591. https://doi.org/10.3324/haematol.2016.147116

32. Mellgren K, Attarbaschi A, Abla O, et al. Non-anaplastic peripheral T cell lymphoma in children and adolescents-an international review of 143 cases. Ann Hematol. 2016; 95(8): 1295-1305. https://doi.org/10.1007/ s00277-016-2722-y

33. Schütte P, Möricke A, Zimmermann M, et al. Preexisting conditions in pediatric ALL patients: Spectrum, frequency and clinical impact. Eur J Med Genet. 2016; 59(3): 143-151. https://doi.org/10.1016/j. ejmg.2015.12.008

34. Dembowska-Baginska B, Perek D, Brozyna A, et al. Non-Hodgkin lymphoma (NHL) in children with Nijmegen Breakage syndrome (NBS). Pediatr Blood Cancer. 2009; 52(2): 186-190. https://doi. org/10.1002/pbc. 21789

35. Fedorova A, Sharapova S, Mikhalevskaya T, et al. Non-Hodgkin Lymphoma in Children with Primary Immunodeficiencies: Clinical Manifestations, Diagnosis, and Management, Belarusian Experience. Lymphoma. 2015; 2015: 1-10. https://doi.org/10.1155/2015/123548

36. Pastorczak A, Szczepanski T, Mlynarski W; International BerlinFrankfurt-Munster (I-BFM) ALL host genetic variation working group. Clinical course and therapeutic implications for lymphoid malignancies in Nijmegen breakage syndrome. Eur J Med Genet. 2016; 59(3): 126-132. https://doi.org/10.1016/j.ejmg.2016.01.007

37. Lehrnbecher T, Averbuch D, Castagnola E, et al. 8th European Conference on Infections in Leukaemia: 2020 guidelines for the use of antibiotics in paediatric patients with cancer or post-haematopoietic cell transplantation. Lancet Oncol. 2021; 22(6): e270-e280. https://doi. org/10.1016/S1470-2045(20)30725-7

38. Groll AH, Pana D, Lanternier F, et al. 8th European Conference on Infections in Leukaemia: 2020 guidelines for the diagnosis, prevention, and treatment of invasive fungal diseases in paediatric patients with cancer or post-haematopoietic cell transplantation. Lancet Oncol. 2021; 22(6): e254-e269. https://doi.org/10.1016/S1470-2045(20)30723-3

39. Bienemann K, Burkhardt B, Modlich S, et al. Promising therapy results for lymphoid malignancies in children with chromosomal breakage syndromes (Ataxia teleangiectasia or Nijmegen-breakage syndrome): a retrospective survey. Br J Haematol. 2011; 155(4): 468-476. https:// doi.org/10.1111/j.1365-2141.2011.08863.x

40. Zawitkowska J, Lejman M, Zaucha-Prażmo A, et al. Grade 3 and 4 Toxicity Profiles During Therapy of Childhood Acute Lymphoblastic Leukemia. In Vivo. 2019; 33(4): 1333-1339. https://doi.org/10.21873/ invivo. 11608

41. Landmann E, Burkhardt B, Zimmermann M, et al. Results and conclusions of the European Intergroup EURO-LB02 trial in children and adolescents with lymphoblastic lymphoma. Haematologica. 2017; 102(12): 2086-2096. https://doi.org/10.3324/haematol.2015.139162

42. Kara B, Koksal Y. Pediatric Lymphoma and Solid Tumors Associated With Cancer Susceptibility Syndromes. J Pediatr Hematol Oncol. 2020; 42(7): 438-445. https://doi.org/10.1097/MPH.0000000000001798

43. Włodarczyk M, Lejman M. Chromosomal instability associated with adverse outcome: a case report of patient with Nijmegen breakage syndrome and rapidly developed T-NHL with complex karyotype. Mol Cytogenet. 2020 Aug 20; 13: 35. https://doi.org/10.1186/s13039020-00505-2. PMID: 32843899; PMCID: PMC7441545.

44. Tomasik B, Pastorczak A, Fendler W, et al. Heterozygous carriers of germline c.657_661del5 founder mutation in NBN gene are at risk of central nervous system relapse of B-cell precursor acute lymphoblastic leukemia. Haematologica. 2018; 103(5): e200-e203. https://doi. org/10.3324/haematol.2017.181198

45. Baleydier F, Bernard F, Ansari M. The Possibilities of Immunotherapy for Children with Primary Immunodeficiencies Associated with Cancers. Biomolecules. 2020; 10(8): 1112. https://doi.org/10.3390/ biom 10081112

46. Kyriakidis I, Vasileiou E, Rossig C, Roilides E, Groll AH, Tragiannidis A. Invasive Fungal Diseases in Children with Hematological Malignancies Treated with Therapies That Target Cell Surface Antigens: Monoclonal Antibodies, Immune Checkpoint Inhibitors and CAR T-Cell Therapies. J Fungi (Basel). 2021; 7(3): 186. https://doi:10.3390/jof7030186

47. Verhoeven D, Stoppelenburg AJ, Meyer-Wentrup F, Boes M. Increased risk of hematologic malignancies in primary immunodeficiency disorders: opportunities for immunotherapy. Clin Immunol. 2018; 190: 22-31. https://doi:10.1016/j.clim.2018.02.007

48. Rubinstein JD, Burns K, Absalon M, et al. EBV-directed viral-specific T-lymphocyte therapy for the treatment of EBV-driven lymphoma in two patients with primary immunodeficiency and DNA repair defects. Pediatr Blood Cancer. 2020; 67(3): e28126. https://doi.org/10.1002/ pbc. 28126

49. June CH, O'Connor RS, Kawalekar OU, Ghassemi S, Milone MC. CAR $\mathrm{T}$ cell immunotherapy for human cancer. Science. 2018; 359(6382): 1361-1365. https://doi:10.1126/science.aar6711

50. Queudeville M, Ebinger M. Blinatumomab in Pediatric Acute Lymphoblastic Leukemia-From Salvage to First Line Therapy (A Systematic Review). J Clin Med. 2021; 10(12):2544. https://doi:10.3390/ jcm10122544

51. Frey N. Cytokine release syndrome: Who is at risk and how to treat. Best Pract Res Clin Haematol. 2017; 30(4): 336-340. https://doi:10.1016/j. beha.2017.09.002

52. Schlegel P, Lang P, Zugmaier G, et al. Pediatric posttransplant relapsed/ refractory B-precursor acute lymphoblastic leukemia shows durable remission by therapy with the T-cell engaging bispecific antibody blinatumomab. Haematologica. 2014; 99(7): 1212-1219. https:// doi:10.3324/haematol.2013.100073

53. 53 Slack J, Albert MH, Balashov D, et al. Outcome of hematopoietic cell transplantation for DNA double-strand break repair disorders. J Allergy Clin Immunol. 2018; 141(1): 322-328.e10. https://doi.org/10.1016/j. jaci.2017.02.036

54. 54 Woźniak M, Krzywoń M, Hołda MK, Goździk J. Reduced-intensity conditioning umbilical cord blood transplantation in Nijmegen breakage syndrome. Pediatr Transplant. 2015; 19(2): E51-E55. https:// doi.org/10.1111/petr.12420

55. Laberko A, Sultanova E, Gutovskaya E, et al. Treosulfan-Based Conditioning Regimen in Haematopoietic Stem Cell Transplantation with TCR $\alpha /$ CD19 Depletion in Nijmegen Breakage Syndrome. J Clin Immunol. 2020; 40(6): 861-871. https://doi.org/10.1007/s10875-02000811-9

56. Gałązka P, Czyżewski K, Szaflarska-Popławska A, Dębski R, Krenska A, Styczyński J. Complex profile of multiple hepatobiliary and gastrointestinal complications after hematopoietic stem cell transplantation in a child with Nijmegen breakage syndrome. Cent Eur J Immunol. 2019; 44(3): 327-331. https://doi.org/10.5114/ceji.2019.89612

57. Moser O, Zimmermann M, Meyer U, et al. Second malignancies after treatment of childhood non-Hodgkin lymphoma: a report of the BerlinFrankfurt-Muenster study group. Haematologica. 2021; 106(5): 13901400. https://doi.org/10.3324/haematol.2019.244780

58. Attarbaschi A, Carraro E, Ronceray L, et al. Second malignant neoplasms after treatment of non-Hodgkin's lymphoma-a retrospective multinational study of 189 children and adolescents. Leukemia. 2021; 35(2): 534-549. https://doi.org/10.1038/s41375-020-0841-x 